\title{
A New Identity for Resolvents of Operators
}

\author{
Michael Gil’ \\ Department of Mathematics, Ben-Gurion University of the Negev, P.O. Box 653, 84105 Beer-Sheva, Israel
}

Correspondence should be addressed to Michael Gil'; gilmi@bezeqint.net

Received 10 December 2012; Accepted 21 December 2012

Academic Editor: Jens Lorenz

Copyright (c) 2013 Michael Gil'. This is an open access article distributed under the Creative Commons Attribution License, which permits unrestricted use, distribution, and reproduction in any medium, provided the original work is properly cited.

\begin{abstract}
A new identity for resolvents of operators is suggested. We show that in appropriate situations it is more convenient than the Hilbert identity. In particular, we establish a new invertibility condition for perturbed operators as well as new bounds for the spectrum of perturbed operators. As a particular case we consider perturbations of Hilbert-Schmidt operators.
\end{abstract}

\section{Introduction and the Main Result}

Let $X$ be a complex Banach space with a norm $\|\cdot\|$ and the unit operator $I$. For a linear operator $A$ in $X,\|A\|=$ $\sup _{x \in X}\|A x\| /\|x\|, \sigma(A)$ is the spectrum, $A^{-1}$ is the inverse operator, and $R_{\lambda}(A)=(A-\lambda I)^{-1}(\lambda \notin \sigma(A))$ is the resolvent.

Everywhere in the following $A$ and $\widetilde{A}$ are bounded operators in $X$, and $E=\widetilde{A}-A$. Recall the Hilbert identity $R_{\lambda}(\widetilde{A})-R_{\lambda}(A)=-R_{\lambda}(A) E R_{\lambda}(\widetilde{A})[1]$. In particular, it gives the following important result: if a $\lambda \in \mathbb{C}$ is regular for $A$ and

$$
\|E\|\left\|R_{\lambda}(A)\right\|<1,
$$

then $\lambda$ is also regular for $\widetilde{A}$. In the present paper we suggest a new identity for resolvents of operators, by which we derive a new invertibility condition for perturbed operators as well as new bounds for the spectrum of perturbed operators. It is shown that in appropriate situations our results improve condition (1). As a particular case we consider perturbations of Hilbert-Schmidt operators.

Put $Z=\widetilde{A} E-E A$. Now we are in a position to formulate and prove our main result.

Theorem 1. Let $a \lambda \in \mathbb{C}$ be regular for $A$ and $\widetilde{A}$. Then

$$
R_{\lambda}(\widetilde{A})-R_{\lambda}(A)=R_{\lambda}(\widetilde{A}) Z R_{\lambda}^{2}(A)-E R_{\lambda}^{2}(A) .
$$

Proof. We have

$$
\begin{aligned}
R_{\lambda}(\widetilde{A}) & (\widetilde{A} E-E A) R_{\lambda}^{2}(A)-E R_{\lambda}^{2}(A) \\
= & {\left[R_{\lambda}(\widetilde{A})(\widetilde{A} E-E A)-E\right] R_{\lambda}^{2}(A) } \\
= & R_{\lambda}(\widetilde{A})[\widetilde{A} E-E A-(\widetilde{A}-\lambda I) E] R_{\lambda}^{2}(A) \\
= & R_{\lambda}(\widetilde{A})(E \lambda-E A) R_{\lambda}^{2}(A)=-R_{\lambda}(\widetilde{A}) E R_{\lambda}(A) \\
= & -R_{\lambda}(\widetilde{A})(\widetilde{A}-\lambda I-(A-\lambda I)) R_{\lambda}(A) \\
= & -\left(I-R_{\lambda}(\widetilde{A})(A-\lambda I)\right) R_{\lambda}(A) \\
= & R_{\lambda}(\widetilde{A})-R_{\lambda}(A),
\end{aligned}
$$

as claimed.

Denote $\eta(A, E, \lambda)=\sup _{0 \leq t \leq 1} t\left\|\left(A E-E A+t E^{2}\right) R_{\lambda}^{2}(A)\right\|$.

Corollary 2. Let a $\lambda \in \mathbb{C}$ be regular for $A$ and $\eta(A, E, \lambda)<1$. Then $\lambda$ is regular also for $\widetilde{A}$.

Indeed, put $A_{t}=A+t E(t \in[0,1])$. Since the regular sets of operators are open, $\lambda$ is regular for $A_{t}$, provided $t$ is small enough. By Theorem 1, we get

$$
\begin{aligned}
R_{\lambda}\left(A_{t}\right)-R_{\lambda}(A)= & R_{\lambda}\left(A_{t}\right)(t(A+t E) E-t E A) R_{\lambda}^{2}(A) \\
& -t E R_{\lambda}^{2}(A) .
\end{aligned}
$$


Hence,

$$
\begin{aligned}
& \left\|R_{\lambda}\left(A_{t}\right)\right\|-\left\|R_{\lambda}(A)-t E R_{\lambda}^{2}(A)\right\| \\
& \quad \leq\left\|R_{\lambda}\left(A_{t}\right)\right\|\left\|\left[t(E A-A E)+t^{2} E^{2}\right] R_{\lambda}^{2}(A)\right\| \\
& \quad \leq\left\|R_{\lambda}\left(A_{t}\right)\right\| \eta(A, E, \lambda) .
\end{aligned}
$$

Thus,

$$
\left\|R_{\lambda}\left(A_{t}\right)\right\| \leq \frac{\left\|R_{\lambda}(A)-t E R_{\lambda}^{2}(A)\right\|}{1-\eta(A, E, \lambda)} .
$$

Taking $t=1$, we obtain the required result.

Furthermore, we have $\eta(A, E, \lambda) \leq \zeta^{2}(A, E)\left\|R_{\lambda}^{2}(A)\right\|$, where

$$
\zeta(A, E):=\sqrt{\|A E-E A\|+\left\|E^{2}\right\|} .
$$

Now Corollary 2 implies.

Corollary 3. If $a \lambda \notin \sigma(A)$ and $\zeta(A, E)\left\|R_{\lambda}(A)\right\|<1$, then $\lambda \notin \sigma(\widetilde{A})$.

Example 4. Let $A=c I$ and $\widetilde{A}=c I+V$ with a nonzero number $c$ and a nilpotent operator $V$ in $X$, such that $V^{2}=0$.

It is clear that $E=V$ and $A$ is invertible. We have $A E-$ $E A=0$ and $\zeta(A, E)=0$. Therefore Corollary 3 gives us the sharp result: $\widetilde{A}$ is invertible for all nonzero $c$.

At the same time (1) gives the invertibility condition $\|V\|<|c|$.

Example 5. Let $X=Y_{1}+Y_{2}$ be a direct sum of two spaces $Y_{1}$ and $Y_{2}$. Besides $Y_{1}=Y_{2}=Y$ is a Banach space with a norm $\|\cdot\|_{Y}$. The norm in $X$ is introduced by $\|x\|=\left\|y_{1}\right\|_{Y}+\left\|y_{2}\right\|_{Y}$, with an $x=y_{1}+y_{2}\left(y_{1} \in Y_{1}, y_{2} \in Y_{2}\right)$. Let us consider the operator matrices

$$
A=\left(\begin{array}{ll}
B & 0 \\
0 & B
\end{array}\right), \quad \widetilde{A}=\left(\begin{array}{ll}
B & C \\
0 & B
\end{array}\right),
$$

where $C$ and $B$ are commuting operators in $Y$. Let $\lambda \notin \sigma(A)$. Again $E=\widetilde{A}-A$. It is simple to check that $E^{2}=0,\|E\|=$ $\|C\|_{Y}$, and $A E-E A=0$. Corollary 3 implies $\lambda \notin \sigma(\widetilde{A})$. At the same time, due to (1), we can assert that $\lambda \notin \sigma(\widetilde{A})$ only if $\|C\|_{Y}\left\|R_{\lambda}(A)\right\|<1$.

Furthermore, following the notions of the matrix perturbation theory, cf. [2], we will say that the spectral variation of $\widetilde{A}$ with respect to $A$ is

$$
s v_{A}(\widetilde{A}):=\sup _{\mu \in \sigma(\widetilde{A})^{s \in \sigma(A)}} \inf _{|\mu-s|,} \mid \mu,
$$

and the Hausdorff distance between the spectra of $A$ and $\widetilde{A}$ is

$$
\operatorname{hd}(A, \widetilde{A}):=\max \left\{s v_{A}(\widetilde{A}), s v_{\widetilde{A}}(A)\right\} \text {. }
$$

\section{Perturbations of Hilbert-Schmidt Operators}

In this section $X=H$ is a separable Hilbert space. Let

$$
N_{2}(A):=\left[\operatorname{Trace}\left(A A^{*}\right)\right]^{1 / 2}<\infty .
$$

That is, $A$ is a Hilbert-Schmidt operator. Introduce the quantity

$$
g(A):=\left[N_{2}^{2}(A)-\sum_{k=1}^{\infty}\left|\lambda_{k}(A)\right|^{2}\right]^{1 / 2} .
$$

The following relations are checked in [3, Section 6.4]:

$$
\begin{gathered}
g^{2}(A) \leq N_{2}^{2}(A)-\mid \text { Trace } A^{2} \mid, \\
g^{2}(A) \leq \frac{N_{2}^{2}\left(A-A^{*}\right)}{2}=2 N_{2}^{2}\left(A_{I}\right),
\end{gathered}
$$

where $A_{I}=\left(A-A^{*}\right) / 2 i$. In our reasonings in the following one can replace $g(A)$ by any of its upper bounds. In particular, one can replace $g(A)$ by $\sqrt{2} N_{2}\left(A_{I}\right)$.

We need the following result.

Theorem 6. Let A be a Hilbert-Schmidt operator. Then

$$
\left\|R_{\lambda}(A)\right\| \leq \sum_{k=0}^{\infty} \frac{g^{k}(A)}{\rho^{k+1}(A, \lambda) \sqrt{k !}} \quad(\lambda \notin \sigma(A)),
$$

where $\rho(A, \lambda)=\inf _{s \in \sigma(A)}|s-\lambda|$, the distance between $\lambda$ and the spectrum of $A$.

For the proof see [3, Theorem 6.4.1]. Now Corollary 3 implies the following.

Corollary 7. If $\lambda$ is regular for $A$, condition (11) holds and

$$
\zeta(A, E) \sum_{k=0}^{\infty} \frac{g^{k}(A)}{\rho^{k+1}(A, \lambda) \sqrt{k !}}<1,
$$

then $\lambda$ is regular for $\widetilde{A}$.

For any $\mu \in \sigma(A)$, due to Corollary 7, we have

$$
\zeta(A, E) \sum_{k=0}^{\infty} \frac{g^{k}(A)}{\rho^{k+1}(A, \mu) \sqrt{k !}} \geq 1 .
$$

Hence it follows that $\rho(A, \mu) \leq x_{0}$, where $x_{0}$ is the unique positive root of

$$
\zeta(A, E) \sum_{k=0}^{\infty} \frac{g^{k}(A)}{x^{k+1} \sqrt{k !}}=1 .
$$

But $s v_{A}(\widetilde{A})=\sup _{\mu \in \sigma(\widetilde{A})} \rho(A, \mu)$. We thus arrive at our next result.

Theorem 8. Let $A$ be a Hilbert-Schmidt operator and $\widetilde{A}$ be an arbitrary bounded operator in $H$. Then $s v_{A}(\widetilde{A}) \leq x_{0}$, where $x_{0}$ is the unique positive root of (17). 
In Section 3 we obtain an estimate for $x_{0}$.

If $A$ is normal, then $g(A)=0$, and consequently $x_{0}=$ $\zeta(A, E)$. Set

Assume that both $A$ and $\widetilde{A}$ are Hilbert-Schmidt operators.

$$
\widehat{g}=\max \{g(A), g(\widetilde{A})\}
$$

Now Theorem 8 implies the following.

Corollary 9. Let both $A$ and $\widetilde{A}$ be Hilbert-Schmidt operators. Then $h d(A, \widetilde{A}) \leq z(\widehat{g})$, where $z(\widehat{g})$ is the unique positive root of the equation

$$
\zeta(A, E) \sum_{k=0}^{\infty} \frac{\widehat{g}^{k}}{x^{k+1} \sqrt{k !}}=1 .
$$

In the following, we suggest an estimate also for $z(\widehat{g})$.

Note that in $[3$, Theorem 8.5 .1$]$, the inequality $s v_{A}(\widetilde{A}) \leq$ $\widehat{x}(\|E\|, A)$ is proved, where $\widehat{x}(\|E\|, A)$ is the unique positive root

$$
\frac{\|E\|}{x} \exp \left[\frac{1}{2}+\frac{g^{2}(A)}{x^{2}}\right]=1 \text {. }
$$

If $E \neq 0$ this inequality gives us a nonzero result. At the same time, if $Z=0$ (as in the above given examples), then Theorem 8 and Corollary 9 give us the sharp result $s v_{A}(\widetilde{A})=$ $\operatorname{hd}(A, \widetilde{A})=0$.

Theorem 6 supplements the recent perturbation results for operators see the interesting papers [4-9] and references given therein.

\section{Estimates for $x_{0}$ and $z(\widehat{g})$}

Denote

$$
\gamma(b, c):=\frac{b \sqrt{2}}{\ln ^{1 / 2}\left[1 / 2+\sqrt{1 / 4+b^{2} / c^{2}}\right]} .
$$

Note that $\gamma(b, c) \rightarrow 0$ as $b \rightarrow 0$ and $c>0$. Similarly, $\gamma(b, c) \rightarrow 0$ as $c \rightarrow 0$ and $b>0$.

Lemma 10. The following inequalities are true:

$$
\begin{gathered}
x_{0} \leq \gamma(g(A), \zeta(A, E)), \\
z(\widehat{g}) \leq \gamma(\widehat{g}, \zeta(A, E)) .
\end{gathered}
$$

Proof. Substituting $x=g(A) y$ into (17), with the notation $q=\zeta(A, E) / g(A)$, we get

$$
1=q \sum_{k=0}^{\infty} \frac{1}{y^{k+1} \sqrt{k !}}
$$

By the Schwarz inequality

$$
\begin{aligned}
\left(\sum_{k=0}^{\infty} \frac{1}{y^{k} \sqrt{k !}}\right)^{2} & =\left(\sum_{k=0}^{\infty} \frac{(\sqrt{2})^{k}}{(\sqrt{2})^{k} y^{k} \sqrt{k !}}\right)^{2} \\
& \leq \sum_{j=0}^{\infty} 2^{-j} \sum_{k=0}^{\infty} \frac{2^{k}}{y^{2 k} k !} \\
& =2 e^{2 / y^{2}}
\end{aligned}
$$

Let $y_{0}=x_{0} / g(A)$ be the unique positive root of (24). Then

$$
1 \leq \frac{q \sqrt{2}}{y_{0}} e^{1 / y_{0}^{2}} \quad \text { or } \quad 1 \leq \frac{2 q^{2}}{y_{0}^{2}} e^{2 / y_{0}^{2}}
$$

and therefore, $y_{0} \leq \hat{y}$, where $\hat{y}$ is the unique positive root of

$$
1=\frac{2 q^{2}}{y^{2}} e^{2 / y^{2}} .
$$

We need the following simple result proved in [10, Lemma 1.6.5].

Lemma 11. The unique positive root $z_{0}$ of the equation

$$
z e^{z}=a \quad(a=\text { const }>0)
$$

satisfies the estimate

$$
z_{0} \geq \ln \left[\frac{1}{2}+\sqrt{\frac{1}{4}+a}\right] .
$$

If, in addition, the condition $a \geq e$ holds, then $z_{0} \geq \ln a-$ $\ln \ln a$.

Put in (27) $z=2 / y^{2}$. Then we obtain (28) with $a=1 / q^{2}$. Now (29) implies

$$
\widehat{y} \leq \frac{\sqrt{2}}{\ln ^{1 / 2}\left[1 / 2+\sqrt{1 / 4+\left(1 / q^{2}\right)}\right]} .
$$

Since $\hat{y} \geq y_{0}=x_{0} / g(A)$ we get inequality (22). Similarly, inequality (23) can be proved.

Now Theorem 8 and Corollary 9 imply the following.

Corollary 12. Let $A$ be a Hilbert-Schmidt operator and $\widetilde{A}$ an arbitrary bounded operator in $H$. Then $s v_{A}(\widetilde{A}) \leq$ $\gamma(g(A), \zeta(A, E))$. If both $A$ and $\widetilde{A}$ are Hilbert-Schmidt operators, then $h d(A, \widetilde{A}) \leq \gamma(\widehat{g}, \zeta(A, E))$.

\section{References}

[1] T. Kato, Perturbation Theory for Linear Operators, SpringerVerlag, New York, NY, USA, 1966.

[2] G. W. Stewart and J. G. Sun, Matrix Perturbation Theory, Academic Press, New York, NY, USA, 1990.

[3] M. I. Gil', Operator Functions and Localization of Spectra, vol. 1830 of Lecture Notes in Mathematics, Springer-Verlag, Berlin, Germany, 2003. 
[4] D. S. Shen, "On the perturbation and continuity of spectralvalued maps for bounded linear operators on Hilbert space," Journal of Mathematical Study, vol. 32, no. 4, pp. 383-389, 1999.

[5] D. J. Kaup and R. A. Van Gorder, "Squared eigenfunctions and the perturbation theory for the nondegenerate $N \times N$ operator: a general outline," Journal of Physics A, vol. 43, no. 43, Article ID 434019, 2010.

[6] C. Perez-Garcia and S. Vega, "Perturbation theory of $p$ Fredholm and semi-Fredholm operators," Koninklijke Nederlandse Akademie van Wetenschappen, vol. 15, no. 1, pp. 115-127, 2004.

[7] P. Aiena, M. González, and A. Martinón, "On the perturbation classes of continuous semi-Fredholm operators," Glasgow Mathematical Journal, vol. 45, no. 1, pp. 91-95, 2003.

[8] W. G. Su and H. J. Zhong, "The generalized West decomposition of operators and other compact perturbation problems," Acta Mathematica Sinica, vol. 22, no. 2, pp. 515-522, 2006.

[9] Y. Xue and G. Chen, "Some equivalent conditions of stable perturbation of operators in Hilbert spaces," Applied Mathematics and Computation, vol. 147, no. 3, pp. 765-772, 2004.

[10] M. I. Gil', Localization and Perturbation of Zeros of Entire Functions, vol. 258 of Lecture Notes in Pure and Applied Mathematics, CRC Press, Boca Raton, Fla, USA, 2010. 


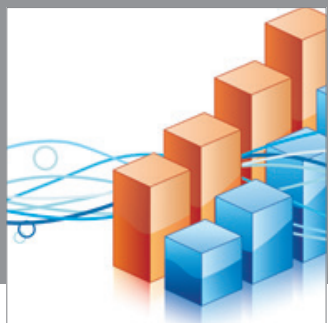

Advances in

Operations Research

mansans

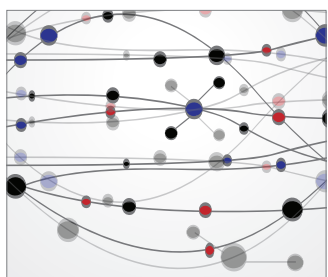

The Scientific World Journal
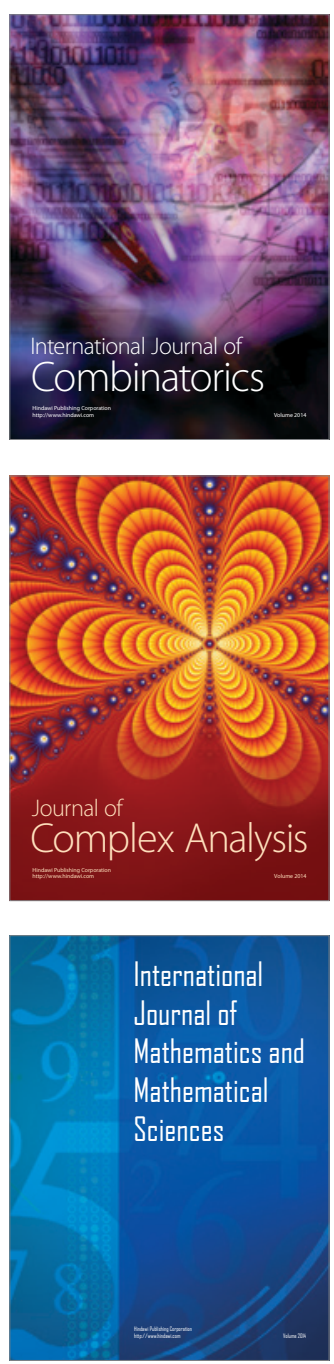
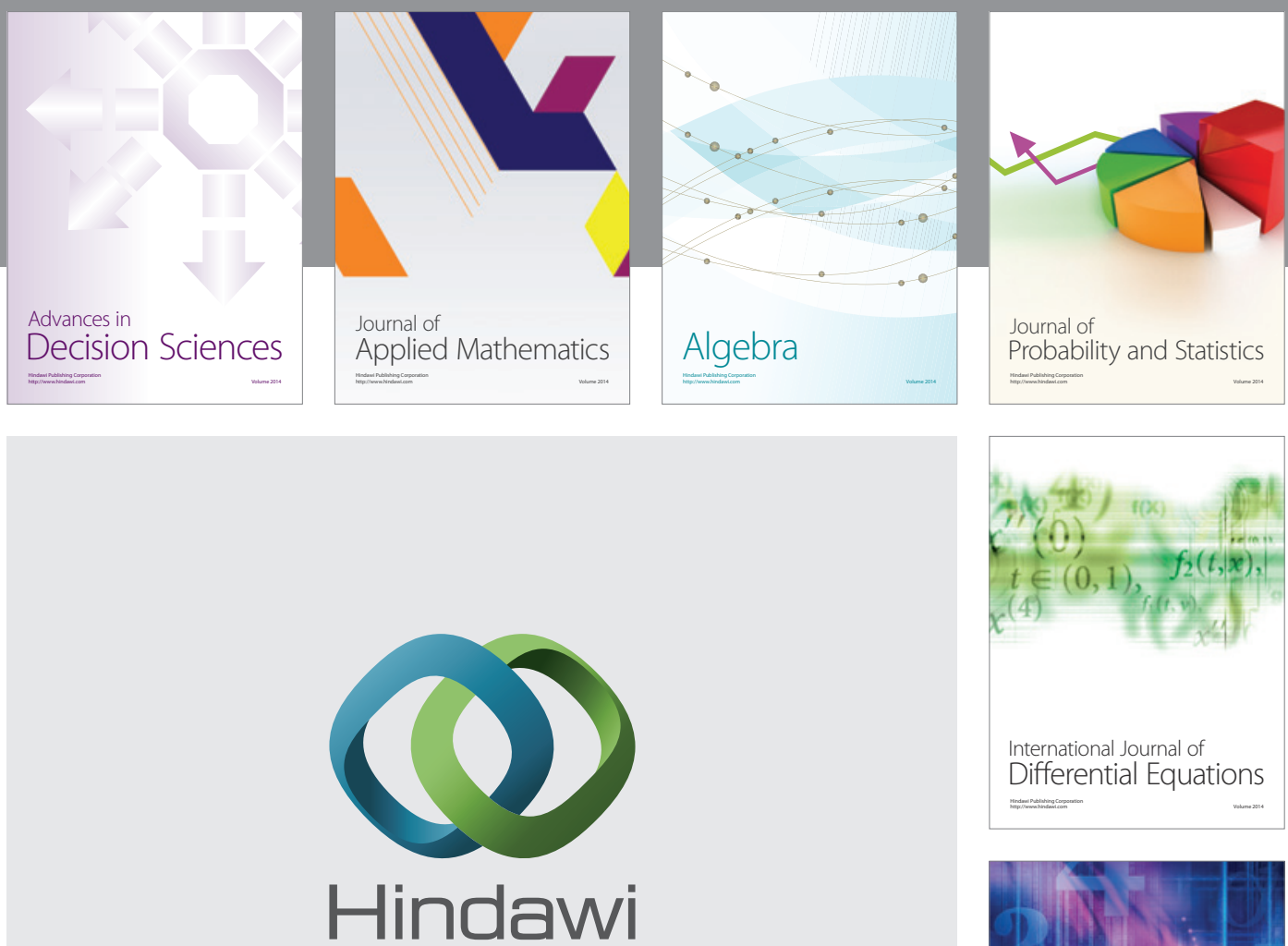

Submit your manuscripts at http://www.hindawi.com
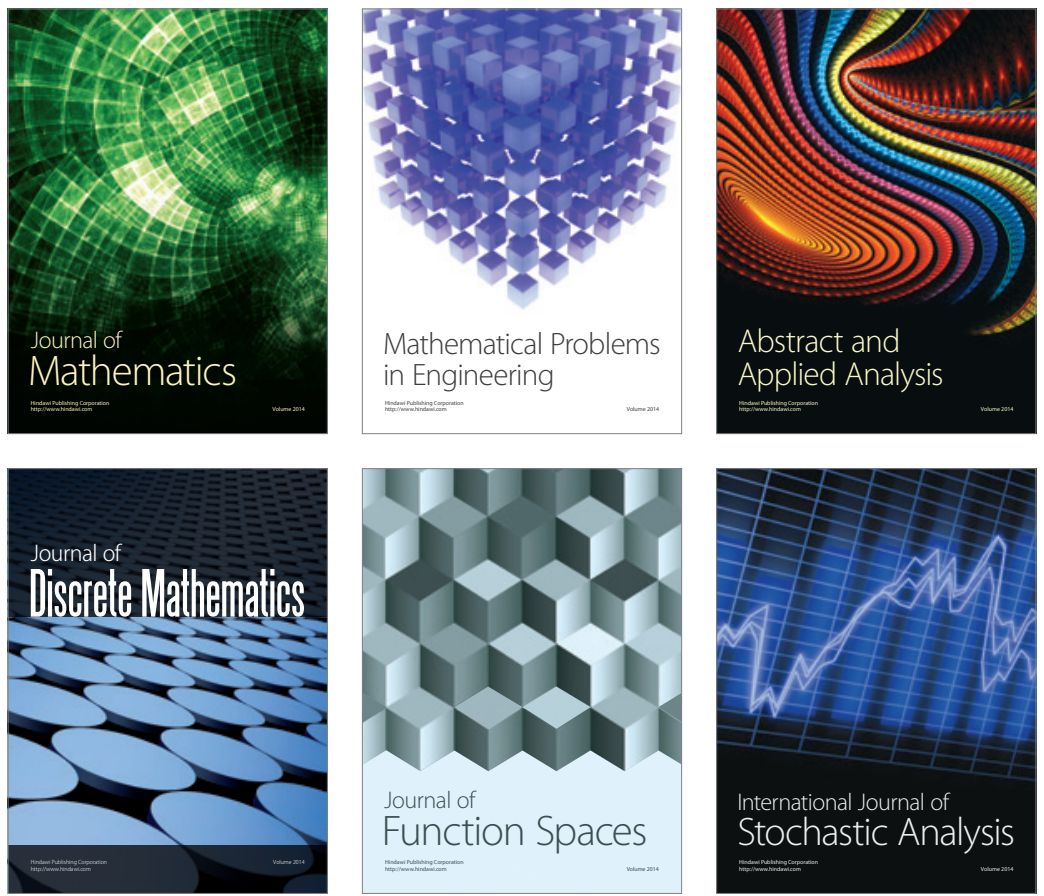

Journal of

Function Spaces

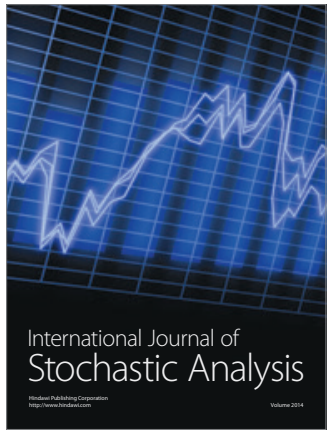

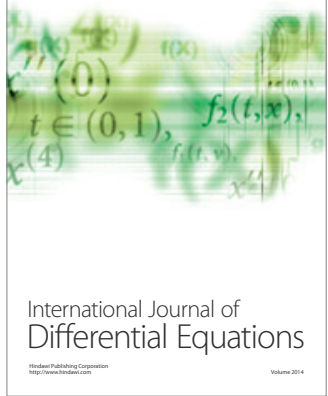
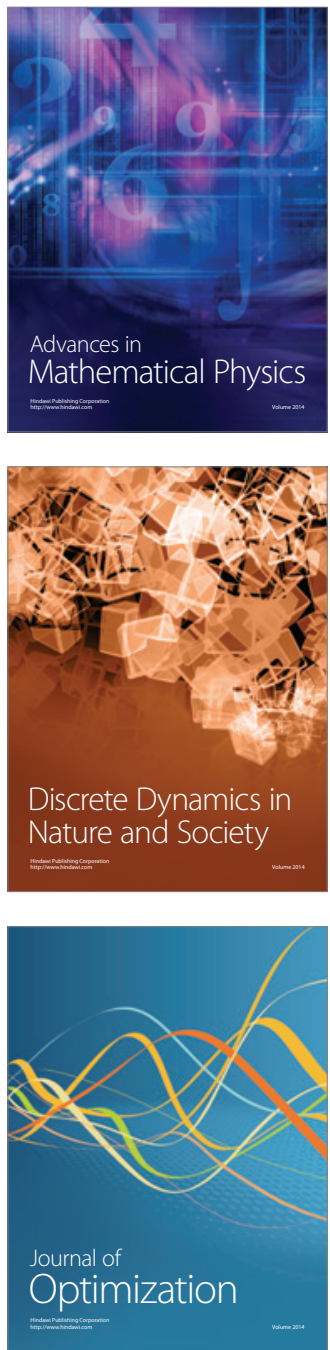